\title{
Prognostic and predictive values of PD-LI expression in patients with digestive system cancer: a meta-analysis
}

This article was published in the following Dove Press journal:

OncoTargets and Therapy

21 July 2017

Number of times this article has been viewed

\section{Cong Dai ${ }^{1, *}$ \\ Meng Wang ${ }^{1, *}$ \\ Jun $\mathrm{Lu}^{2}, *$ \\ Zhiming Dai ${ }^{3}$ \\ Shuai Lin' \\ Pengtao Yang' \\ Tian Tian' \\ Xinghan Liu' \\ Weili Min' \\ Zhijun Dai' \\ 'Department of Oncology, The Second Affiliated Hospital of Xi'an Jiaotong University, ${ }^{2}$ Clinical Research Center, The First Affiliated Hospital of Xi'an Jiaotong University, ${ }^{3}$ Department of Anesthesia, The Second Affiliated Hospital of Xi'an Jiaotong University, Xi'an, People's Republic of China}

*These authors contributed equally to this work
Correspondence: Zhijun Dai Department of Oncology, The Second Affiliated Hospital of Xi'an Jiaotong University, No 157, Xiwu Road, Xi'an City, Shanxi Province, People's Republic of China 710004

Tel +862987679519

Email dzj09।I@I26.com
Background: PD-L1 has been reported to be expressed in diverse human malignancies. However, the prognostic value of PD-L1 in digestive system cancers remains inconclusive. Therefore, we conducted this meta-analysis to evaluate the prognostic impact of PD-L1 expression in digestive system cancers.

Materials and methods: We searched the PubMed, Embase, and the Chinese National Knowledge Infrastructure for publications concerning PD-L1 expression in digestive system cancers. Correlations of PD-L1 expression level with overall survival (OS), disease-free survival (DFS), and recurrence-free survival (RFS) were analyzed.

Results: Finally, 32 studies with 7,308 patients were included. Our results show that PD-L1 expression was significantly associated with poorer OS (hazard ratio $[\mathrm{HR}]=1.44,95 \%$ confidence interval $[\mathrm{CI}]=1.18-1.76, P<0.001)$, but not DFS (HR $=0.91,95 \% \mathrm{CI}=0.61-1.37, P=0.657$ ) or RFS (HR $=1.27,95 \% \mathrm{CI}=0.75-2.14, P=0.368)$. Moreover, in the subgroup analysis, significant associations between PD-L1 expression and OS were found in Asians (HR $=1.50,95 \%$ $\mathrm{CI}=1.19-1.89, P=0.001)$, gastric cancer $(\mathrm{HR}=1.43,95 \% \mathrm{CI}=1.05-1.94, P=0.021)$, and pancreatic carcinoma $(\mathrm{HR}=2.64,95 \% \mathrm{CI}=1.78-3.93, P<0.001)$.

Conclusion: These results suggest that the expression of PD-L1 is associated with worse OS in digestive system cancers, especially in gastric cancer and pancreatic cancer. In addition, PD-L1 may act as a new parameter for predicting poor prognosis and a promising target for anticancer therapy in digestive system cancers.

Keywords: PD-L1, digestive system cancers, prognosis, meta-analysis

\section{Introduction}

Cancer is now the major cause of death in developed countries, and its incidence and mortality are increasing for several cancer types, among which the most fatal are liver and pancreatic cancer. ${ }^{1}$ Liver and pancreatic cancer are digestive system cancers, which also includes esophageal cancer (EC), gastric cancer, biliary tract cancer, and colorectal cancer (CRC). Of all the cancers, digestive system cancers demonstrate the highest incidence and death rates. ${ }^{2,3}$ Recently, the development of multidisciplinary therapies has significantly improved treatment outcomes, but the overall prognosis for sufferers of digestive system cancers is still poor. Current research is increasingly focused on new immunotherapeutic strategies, which could be a major breakthrough in the field of cancer treatment. ${ }^{4}$ In addition, certain immunologic checkpoint markers have been reported in digestive system cancers, among which PD-L1 is the focus of studies. ${ }^{5}$ 
PD-L1, also known as CD274 and B7-H1, is a member of the B7 family of immune regulatory cell surface proteins. ${ }^{6}$ It is commonly upregulated in many different human tumors. The combination of PD-L1 with the receptor PD-1, which has been reported to form and maintain an immunosuppressive microenvironment by suppressing the proliferation of activated T-cells and inducing the apoptosis of T-cells, is considered to be an important immunological escape mechanism that increases the risk of neoplasia. ${ }^{7-10}$ In addition, immune checkpoint blockade using PD-L1 antibodies seems to be one of the most promising immunotherapy approaches. ${ }^{11}$ Although anti-PD-L1 therapies are continuously developing, the prognostic value of PD-L1 is still unclear in various digestive system cancers.

There are many studies that demonstrate the relationship between PD-L1 and survival of patients with different digestive system cancers. But, the conclusions have not reached a consensus and most studies only focused on one cancer type, and did not make an assessment on digestive system cancers. Herein, we conducted a meta-analysis to assess the impact of PD-L1 on the prognosis of digestive system cancers.

\section{Methods}

This meta-analysis complied with the Preferred Reporting Items for Systematic Reviews and Meta-Analyses (PRISMA) guidelines.

\section{Literature search strategy}

We searched the PubMed, Embase, and the Chinese National Knowledge Infrastructure databases (up to June 2016) to obtain the relevant articles. The following keywords were used: "PD-L1," "B7-H1," "CD274," "digestive system cancer," "colorectal neoplasms," "esophageal neoplasms," "gastric cancer," "hepatocellular carcinomas," "pancreatic carcinomas," "biliary tract neoplasms," and "prognosis." We also searched the references of the included studies manually to identify relevant publications. Two authors (Cong Dai and Meng Wang) performed the search strategy, and discrepancies were discussed by all the researchers in the group meeting.

\section{Inclusion and exclusion criteria}

No language restrictions were applied. If different studies published by the same investigators have overlapping data, only the most complete one was included.

Studies were included if they fulfilled the following criteria: 1) all selected cancer cases were pathologically confirmed; 2) studies evaluated the relationship of PD-L1 expression in digestive system cancer patients with detailed information of overall survival (OS), disease-free survival (DFS), or recurrence-free survival (RFS); and 3) the study provided a hazard ratio (HR) with the corresponding confidence interval (CI) or sufficient data to calculate it. Articles meeting the following criteria were excluded: 1) unrelated or duplicate publication; 2) nonhuman experiments were performed; 3) case series, case reports, reviews, or studies without original data; 4) crude data were not provided or HRs could not be calculated.

\section{Data extraction}

Two independent researchers (Zhiming Dai and Shuai Lin) extracted the detailed information of included studies with a standardized format. The results were compared, and all the researchers in the group meeting discussed the final decision in case of any discrepancy. The following information was collected: first author surname, year of publication, patient source, number of patients, tumor types, specimen types, method of detection, PD-L1 expression, median follow-up, prognostic outcomes, HR estimate, and HR with its 95\% CI. If any of these data were not offered in the study, items were recorded as "-".

\section{Quality assessment}

Methodological quality was assessed using the NewcastleOttawa Scale (NOS). The scale includes three domains: selection, comparability, and outcome assessment. Studies with a score of 6-9 were regarded as high quality. Two authors (Cong Dai and Meng Wang) independently graded each study, and all the researchers in the group met to make final decisions regarding any discrepancies.

\section{Statistical analyses}

The statistical analyses were performed with Stata 12.0 (StataCorp LP, College Station, TX, USA). We computed the pooled HR and its $95 \% \mathrm{CI}$ to evaluate the relationship between the PD-L1 and the prognosis of patients with digestive system cancer. In addition, prognostic markers were classified into OS, DFS, and RFS. If HRs were provided explicitly in the studies, we used them directly. Otherwise, we calculated the HR from the Kaplan-Meier survival curve or with the available data using methods described by Parmer et al. ${ }^{12}$ Data from the Kaplan-Meier survival curves were read by Engauge Digitizer version 4.1. The $Q$ test and the $I^{2}$ test were used to evaluate the heterogeneity among studies. If heterogeneity was significant $\left(P<0.1\right.$ or $\left.I^{2}>50 \%\right)$, 
random-effects model was used. ${ }^{13}$ Otherwise, a fixed-effects Mantel-Haenszel model was applied. ${ }^{14}$ We further conducted subgroup analyses by ethnicity, tumor type, and HR estimate. Sensitivity analysis was performed by omitting individual studies to examine the reliability of the results. Meta-regression analysis was conducted to identify potential factors causing heterogeneity. ${ }^{15}$ Publication bias was assessed using Begg's test, Egger's test, and Begg's funnel plot. ${ }^{16,17}$

\section{Results}

\section{Characteristics of included studies}

We initially identified a total of 343 studies using the search criteria listed earlier. As shown in Figure 1, 311 studies were excluded owing to irrelevance to the analysis or lack of the relevant data we needed, or because they were reviews, letters, or animal experiments. Finally, there were 32 studies included in this meta-analysis. ${ }^{18-49}$

We have summarized the characteristics of the 32 studies in Table 1. Of the 32 publications, 30 assessed the relationship between PD-L1 and OS in patients with digestive system neoplasms. In addition, eight studies evaluated the relationship between PD-L1 and DFS, and three studies evaluated PD-L1 and RFS. Studies with a total of 7,308 patients, from China, Korea, Japan, the UK, Switzerland, and Germany, were enrolled. In addition, the number of patients in each study ranged from 40 to 1,420 . To observe the status of PD-L1 in patients with different cancers, we categorized the cancers into CRC (six studies), EC (five studies), gastric cancer (13 studies), hepatocellular cancer (four studies), pancreatic

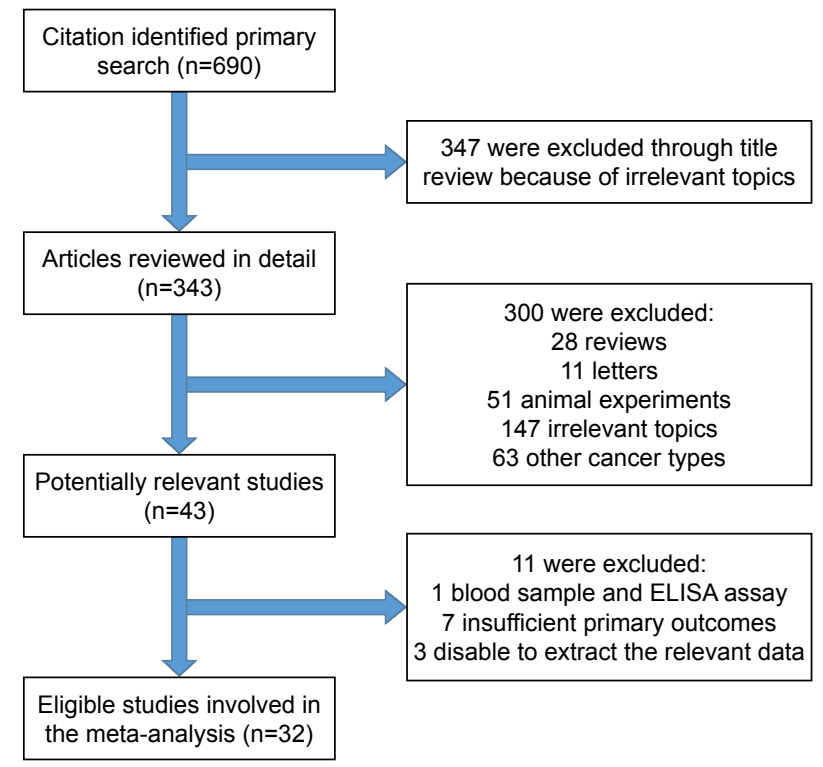

Figure I Flowchart of the selection of the studies in the meta-analysis. Abbreviation: ELISA, enzyme-linked immunoabsorbent assay. cancer (three studies), and extrahepatic bile duct cancer (one study). The median positive rate of PD-L1 was $49.6 \%$ (range $19.8 \%-84.8 \%$ ). The median follow-up times ranged from 20.7 to 75 months. In addition, in methodological quality assessment, all of the studies, which obtained scores ranging from 6 to 9, were considered high quality (Table 1).

\section{Main meta-analysis results}

Overall, there were 30 studies including 6,801 patients concerning the association between OS and PD-L1 expression. The meta-analysis results show that positive expression was associated with significantly poorer OS compared to the negative expression $(\mathrm{HR}=1.44,95 \% \mathrm{CI}=1.18-1.76$, $P<0.001$; Table 2 and Figure 2). The heterogeneity among studies was statistically significant $\left(P<0.001, I^{2}=87.8 \%\right)$; therefore, a random-effects model was used.

Three studies with 544 patients reported the RFS. Owing to the significant heterogeneity $\left(P=0.023, I^{2}=68.5 \%\right)$ among these studies, a random-effects model was used. Our results suggested that PD-L1 was not associated with RFS of digestive system cancers $(\mathrm{HR}=1.27,95 \% \mathrm{CI}=0.75-2.14$, $P=0.368$; Figure 3).

As shown in Table 3 and Figure 4, there were eight studies comprising 1,566 patients which provided results regarding DFS and PD-L1 expression. The pooled data demonstrated that there was no association between them $(\mathrm{HR}=0.91,95 \%$ $\mathrm{CI}=0.61-1.37, P=0.657$, random-effects model $)$.

\section{Subgroup analyses, sensitivity analyses, and meta-regression in OS}

To solve the heterogeneity, we performed subgroup analyses by ethnicity, tumor types, and HR estimate. The subgroup analysis by ethnicity suggested a significant association in studies based on Asians ( $\mathrm{HR}=1.50,95 \% \mathrm{CI}=1.19-1.89$, $P=0.001)$ but not among other ethnicities ( $\mathrm{HR}=1.07,95 \%$ $\mathrm{CI}=0.72-1.58, P=0.740)$. In the subgroup analysis by tumor types, significant associations were found in gastric cancer (HR $=1.43,95 \% \mathrm{CI}=1.05-1.94, P=0.021)$ and pancreatic carcinoma $(\mathrm{HR}=2.64,95 \% \mathrm{CI}=1.78-3.93, P<0.001)$. For HR estimation, subgroup analysis showed that the overall HR estimate with univariate analysis was $1.64(95 \%$ $\mathrm{CI}=1.21-2.23, P=0.001$; Table 2). Meanwhile, it is worth mentioning that heterogeneity among most of the subgroups was statistically significant $\left(P>0.1\right.$ or $\left.I^{2}>50 \%\right)$.

The included studies were sequentially removed to investigate whether any single study has an influence on the pooled results. As shown in Figure 5, the stable pooled HR was not significantly affected by any individual study. 


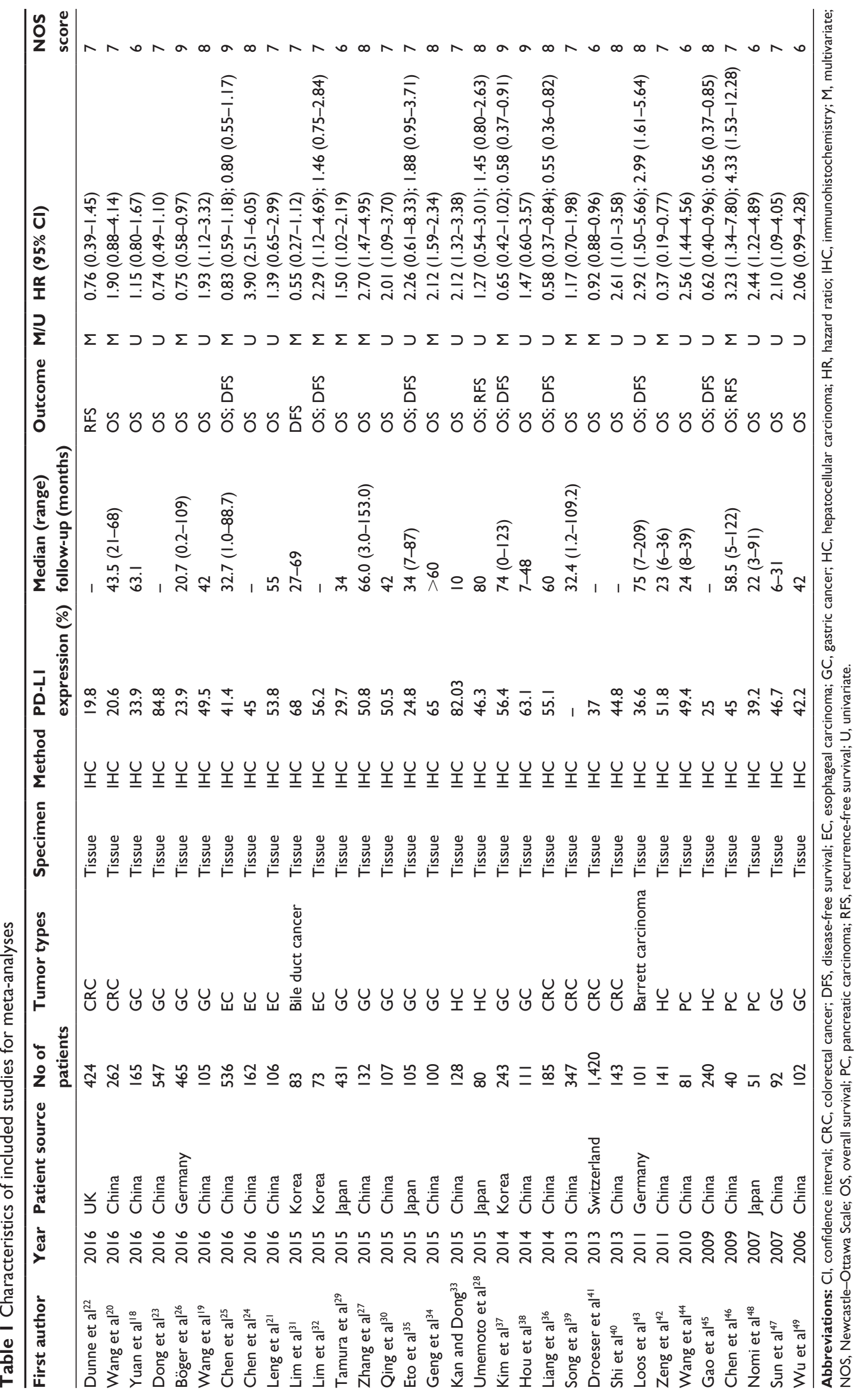


Table 2 Main meta-analysis results for OS

\begin{tabular}{|c|c|c|c|c|c|c|c|}
\hline \multirow[t]{2}{*}{ Analysis } & \multirow{2}{*}{$\begin{array}{l}\text { No of } \\
\text { studies }\end{array}$} & \multirow{2}{*}{$\begin{array}{l}\text { No of } \\
\text { patients }\end{array}$} & \multirow[t]{2}{*}{ Model } & \multirow[t]{2}{*}{ HR (95\% Cl) } & \multirow[t]{2}{*}{$P$-value } & \multicolumn{2}{|c|}{ Heterogeneity } \\
\hline & & & & & & $I^{2}(\%)$ & $P$-value \\
\hline OS & 30 & 6,801 & Random & $1.44(1.18-1.76)$ & 0.000 & 87.8 & 0.000 \\
\hline \multicolumn{8}{|l|}{ Ethnicity } \\
\hline Asian & 27 & 4,815 & Random & $1.50(1.19-1.89)$ & 0.001 & 82.0 & 0.000 \\
\hline Non-Asian & 3 & 1,98 & Random & $1.07(0.72-1.58)$ & 0.740 & 85.7 & 0.001 \\
\hline \multicolumn{8}{|l|}{ Tumor types } \\
\hline CRC & 5 & 2,357 & Random & $1.15(0.76-1.72)$ & 0.511 & 79.7 & 0.001 \\
\hline GC & 13 & 2,705 & Random & $1.43(1.05-1.94)$ & 0.021 & 83.6 & 0.000 \\
\hline EC & 5 & 978 & Random & $1.96(0.97-3.97)$ & $0.06 \mathrm{I}$ & 88.1 & 0.000 \\
\hline $\mathrm{HC}$ & 4 & 589 & Random & $0.89(0.40-1.97)$ & 0.776 & 86.5 & 0.000 \\
\hline PC & 3 & 172 & Fixed & $2.64(1.78-3.93)$ & 0.00 & 0.0 & 0.878 \\
\hline \multicolumn{8}{|l|}{ HR estimate } \\
\hline Multivariate analysis & 12 & 4,190 & Random & $1.22(0.92-1.63)$ & 0.171 & 90.5 & 0.000 \\
\hline Univariate analysis & 18 & 2,611 & Random & $1.64(I .2 \mid-2.23)$ & 0.001 & 80.4 & 0.000 \\
\hline
\end{tabular}

Note: Bold means that there was significant association between PD-LI expression and the items of "Analysis".

Abbreviations: $\mathrm{Cl}$, confidence interval; CRC, colorectal cancer; EC, esophageal carcinoma; GC, gastric cancer; HC, hepatocellular carcinoma; HR, hazard ratio; OS, overall survival; PC, pancreatic carcinoma.

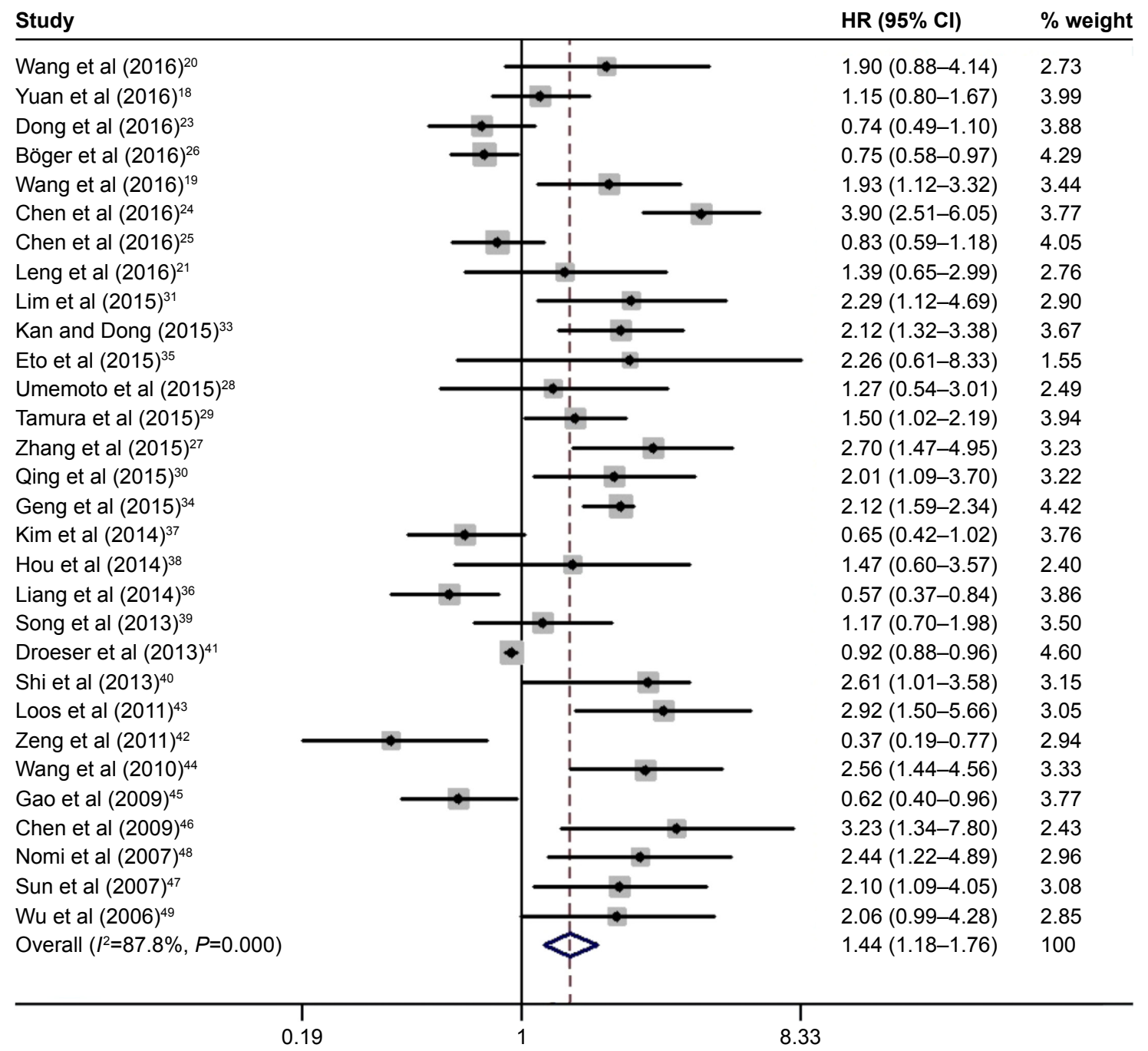

Figure 2 Forest plot of HR for the association of PD-LI overexpression and OS.

Note: Weights are from random-effects analysis.

Abbreviations: $\mathrm{Cl}$, confidence interval; $\mathrm{HR}$, hazard ratio; OS, overall survival. 


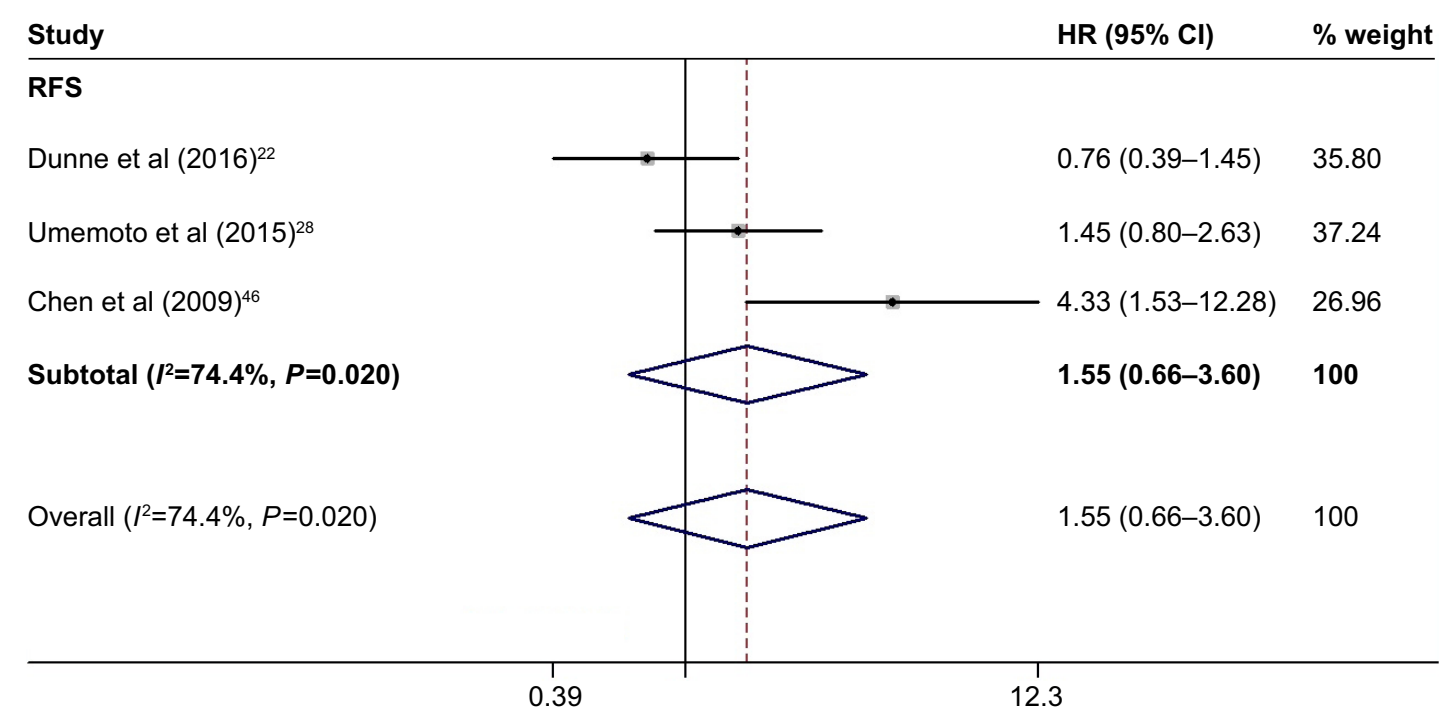

Figure 3 Forest plot of HR for the association of PD-LI overexpression and RFS.

Note: Weights are from random-effects analysis.

Abbreviations: $\mathrm{Cl}$, confidence interval; $\mathrm{HR}$, hazard ratio; RFS, recurrence-free survival.

The meta-regression was performed to identify the source of the heterogeneity. We analyzed possible factors including publication year, ethnicity, number of patients, tumor types, and PD-L1 expression. The results confirmed that the number of patients per study might be a major source of heterogeneity (Table 4).

\section{Subgroup analyses in DFS}

We also performed subgroup analysis for ethnicity, tumor types, and HR estimate among studies focus on DFS. Only the subgroup analysis for the HR estimate showed a significant association with DFS based on multivariate analysis (HR $=0.75,95 \% \mathrm{CI}=0.59-0.97, P=0.026$; Table 3$)$.

\section{Publication bias}

We performed Begg's and Egger's tests to identify whether any publication bias existed in the published literature in this meta-analysis. Publication bias was observed among studies reporting OS ( $P=0.498,0.003)$, but no publication bias was found among studies reporting DFS $(P=0.230,0.330)$ or RFS $(P=0.308,0.328)$. The Begg's plots for the effect of PD-L1 expression on OS are shown in Figure 6.

\section{Discussion}

Recently, according to many reports, cancer cells are able to use immunosuppressive molecules to their advantage via inhibiting antitumor lymphocytes, thus evading destruction by the immune system. Therefore, immunotherapy is now considered a novel method of cancer treatment. ${ }^{50}$ Cancer immunotherapy is a revolutionary cancer treatment targeting the immune checkpoint receptors such as PD-L1. PD-L1 antibodies have been proved to exert clinical activity in more than 15 types of cancers including EC, gastric cancer, hepatocellular cancer, and CRC. ${ }^{51}$

Table 3 Main meta-analysis results for DFS

\begin{tabular}{|c|c|c|c|c|c|c|c|}
\hline \multirow[t]{2}{*}{ Analysis } & \multirow{2}{*}{$\begin{array}{l}\text { No of } \\
\text { studies }\end{array}$} & \multirow{2}{*}{$\begin{array}{l}\text { No of } \\
\text { patients }\end{array}$} & \multirow[t]{2}{*}{ Model } & \multirow[t]{2}{*}{ HR (95\% Cl) } & \multirow[t]{2}{*}{$P$-value } & \multicolumn{2}{|c|}{ Heterogeneity } \\
\hline & & & & & & $I^{2}(\%)$ & $P$-value \\
\hline DFS & 8 & 1,566 & Random & $0.91(0.61-1.37)$ & 0.657 & 80.1 & 0.000 \\
\hline \multicolumn{8}{|l|}{ Ethnicity } \\
\hline Asian & 7 & 1,465 & Random & $0.76(0.56-1.05)$ & 0.094 & 64.2 & 0.010 \\
\hline Non-Asian & 1 & 101 & - & $0.97(0.62-1.52)$ & 0.001 & - & - \\
\hline \multicolumn{8}{|l|}{ Tumor types } \\
\hline GC & 2 & 348 & Random & I.0I (0.32-3.2I) & 0.981 & 87.5 & 0.005 \\
\hline EC & 3 & 710 & Random & $1.47(0.66-3.30)$ & 0.346 & 84.5 & 0.002 \\
\hline \multicolumn{8}{|l|}{ HR estimate } \\
\hline Multivariate analysis & 4 & 935 & Fixed & $0.75(0.59-0.97)$ & 0.026 & 49.5 & 0.115 \\
\hline Univariate analysis & 4 & 631 & Random & I.II (0.50-2.46) & 0.805 & 89.6 & 0.000 \\
\hline
\end{tabular}

Note: Bold means that there was significant association between PD-LI expression and the items of "Analysis".

Abbreviations: $\mathrm{Cl}$, confidence interval; DFS, disease-free survival; EC, esophageal carcinoma; GC, gastric cancer; HR, hazard ratio. 


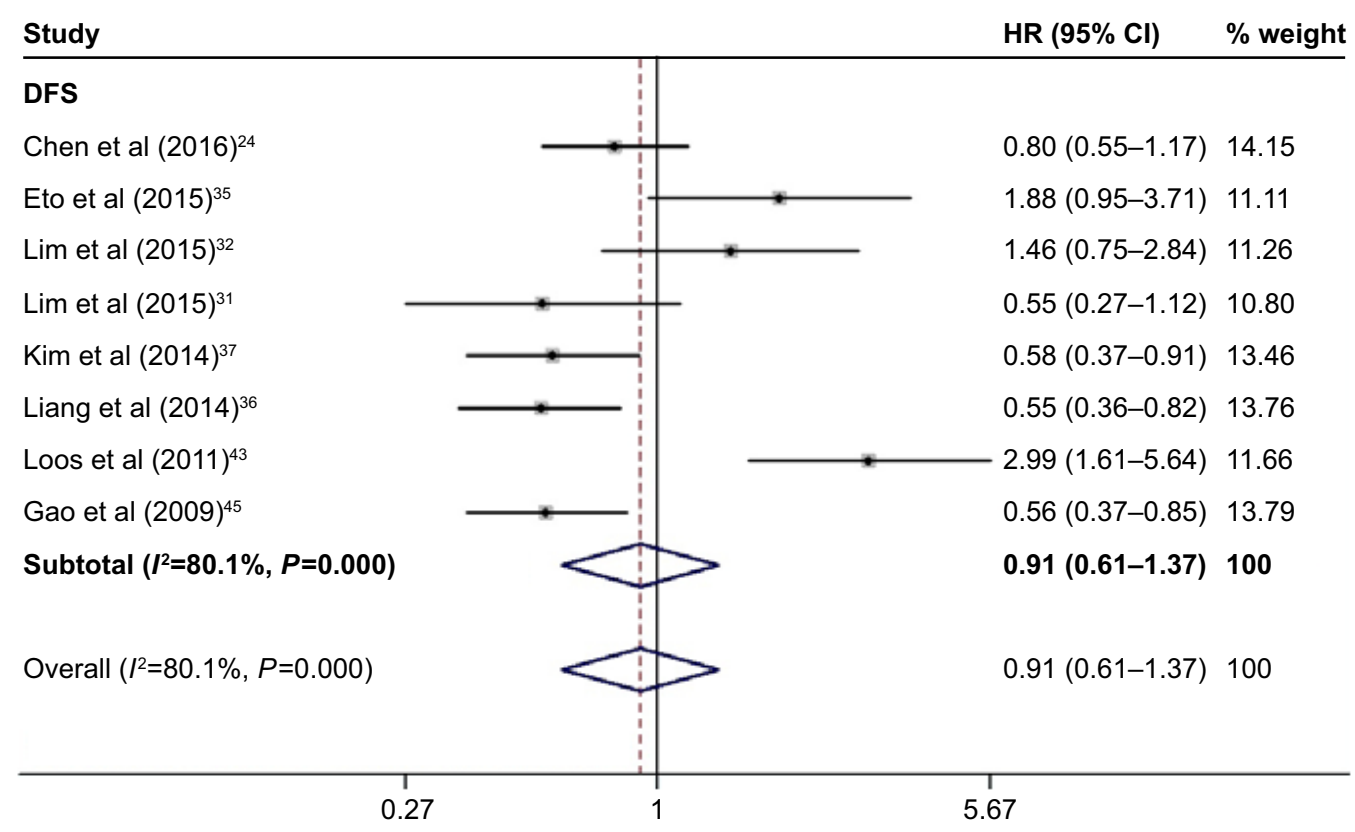

Figure 4 Forest plot of HR for the association of PD-LI overexpression and DFS.

Note: Weights are from random-effects analysis.

Abbreviations: $\mathrm{Cl}$, confidence interval; DFS, disease-free survival; $\mathrm{HR}$, hazard ratio.

These studies suggested that PD-L1 may be a prognostic biomarker and a potential target of treatment in digestive system cancers. In this meta-analysis, we investigated the association between the expression of PD-L1 and the prognosis of digestive system cancer patients by analyzing the published data.

Our results showed that PD-L1 overexpression was significantly associated with shorter OS. These results indicated

\section{Meta-analysis estimates, given named study is omitted}

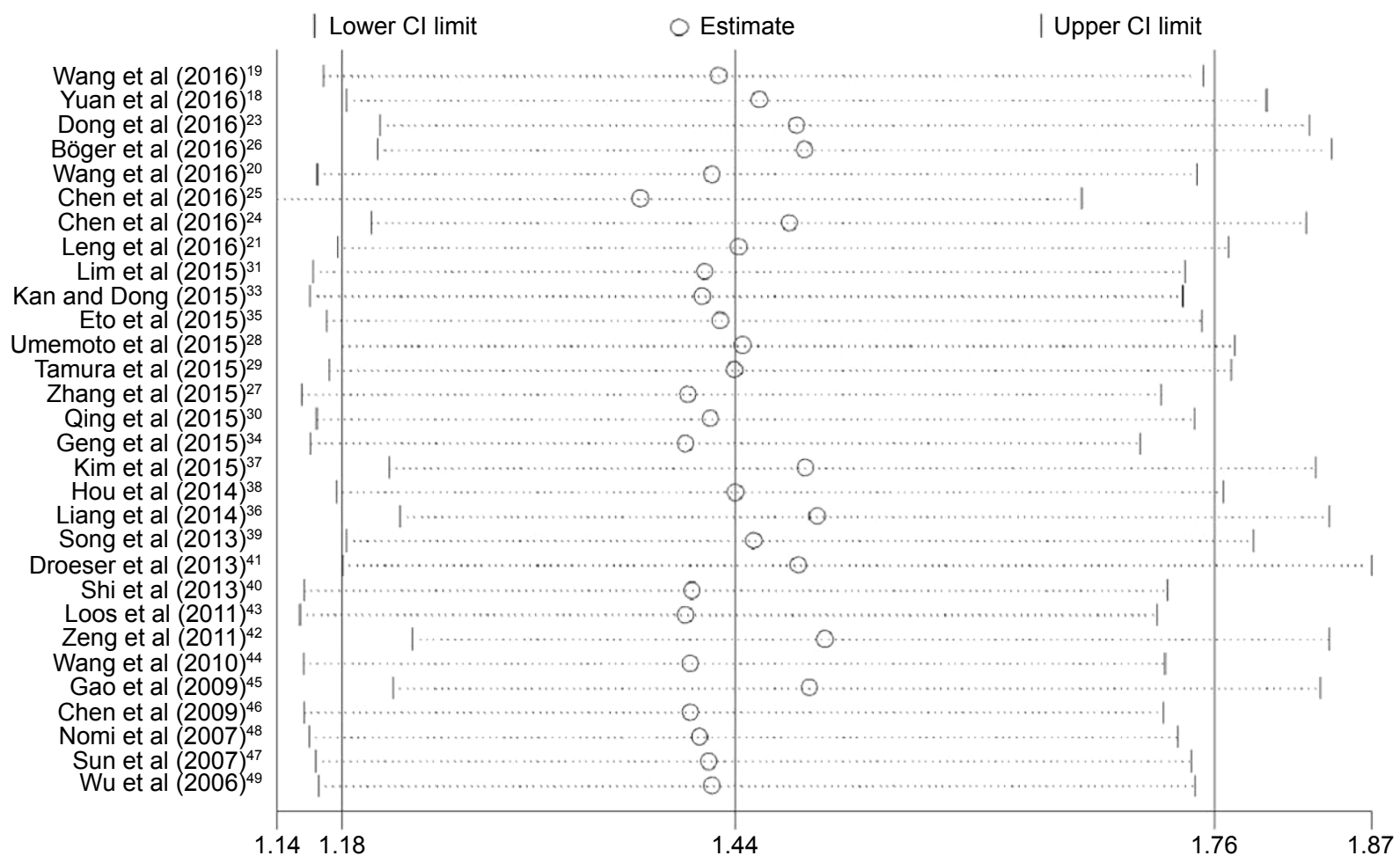

Figure 5 Sensitivity analysis of pooled HRs on the association between PD-LI expression and OS.

Abbreviations: $\mathrm{Cl}$, confidence interval; $\mathrm{HR}$, hazard ratio; OS, overall survival. 
Table 4 Meta-regression analyses of potential source of heterogeneity

\begin{tabular}{lllllll}
\hline Heterogeneity factors & Coefficient & SE & Z & P-value & \multicolumn{2}{c}{$\mathbf{9 5 \% \text { CI }}$} \\
\hline Publication year & & & & LL & UL \\
Ethnicity & -0.024 & 0.038 & -0.63 & 0.536 & -0.102 & 0.054 \\
Number of patients & -0.246 & 0.351 & -0.70 & 0.489 & -0.966 & 0.473 \\
Tumor types & -0.001 & 0.000 & -2.33 & 0.028 & -0.002 & -0.000 \\
PD-LI expression & 0.089 & 0.094 & 0.94 & 0.353 & -1.039 & 0.282 \\
\hline
\end{tabular}

Abbreviations: $\mathrm{Cl}$, confidence interval; LL, lower limit; SE, standard error; UL, upper limit.

that PD-L1 can serve as a novel parameter for prognostication and a promising target for anticancer therapy in digestive system cancers. When extended to subgroup analysis, no clear correlation between PD-L1 expression and OS was found in non-Asian, $\mathrm{CRC}, \mathrm{EC}$, and hepatocellular carcinoma (HC) patients, possibly owing to the insufficiently large sample size. Therefore, it is necessary for better designed studies with more patients to prove or to retort our results in the future.

There was a significant heterogeneity across the included studies. However, subgroup analyses of ethnicity, tumor types, and the methods used to estimate the HR failed to identify its source. The sensitivity analyses showed that the stable pooled HR was not significantly affected by each individual study. The meta-regression confirmed that the number of patients might be a major source of heterogeneity. However, the meta-regression adjusted $R^{2}$ value of the number of patients is just $15.78 \%$ (data not shown), which could only explain a small part of heterogeneity source. Therefore, it is likely that the heterogeneity is due to the differences in the baseline characteristics of patients, the immunohistochemical methods, and the baseline referring to positive/high PD-L1 expression. However, because of lacking clinical data in

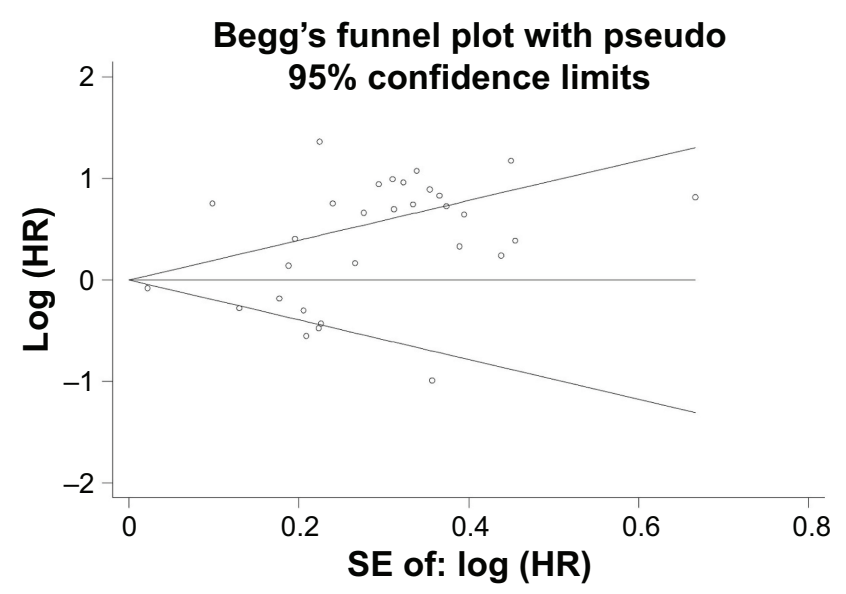

Figure 6 Funnel plots of publication bias for all the included studies reported with OS.

Abbreviations: HR, hazard ratio; OS, overall survival; SE, standard error. these aspects, we cannot determine their contribution to the heterogeneity among studies.

Previously, some meta-analyses have been conducted to study the association between PD-L1 and some cancers in the digestive system. Huang et $\mathrm{al}^{52}$ identified nine studies that involved 2,500 gastrointestinal tract cancer patients, and reported that PD-L1 is a prognostic risk factor for gastrointestinal tract cancer. The study by Wu et al, ${ }^{53}$ which included seven studies with 687 patients, showed that positive PD-L1 expression status in tumor cells was associated with worse 5-year OS of EC, gastric cancer, and CRC. Compared with these studies, our meta-analysis has some differences and advantages. First of all, our study is the first meta-analysis to estimate the role of PD-L1 in the prognosis of digestive system cancers. Second, we not only analyzed OS but also evaluated DFS and RFS, which contributed to a comprehensive understanding of the issue. Third, our conclusion is more reliable because several new studies fulfilling the inclusion criteria have been included.

We attempted to conduct a comprehensive analysis of PD-L1 and prognosis of digestive system cancers, but it should be recognized that there are still some limitations in our study. First, some survival data were not reported directly; therefore, an indirect method was used to extract data from the Kaplan-Meier survival curves, which may affect the accuracy of the original data. Second, publication bias was observed among studies reporting OS. The reason may be that studies with positive results were published more easily. Third, we cannot recognize the association between subgroups of RFS and PD-L1 expression due to lack of data. More importantly, the PD-L1 expression level measured using immunohistochemistry might show high variability between studies. Hence, a baseline for positive/high PD-L1 expression should be established.

\section{Conclusion}

We found that PD-L1 expression indicated poor OS outcomes. We indicated that PD-L1 may serve as a prognostic 
indicator and a potential novel target for treatment in digestive system cancers.

\section{Acknowledgments}

This study was supported by the National Natural Science Foundation, China (No 81471670); China Postdoctoral Science Foundation (No 2014M560791; 2015T81037); the Fundamental Research Funds for the Central Universities, China (No 2014qngz-04); the International Cooperative Project of Shaanxi province, People's Republic of China (No 2014KW-23-07), and Science and Technology Plan of Innovation Project, Shaanxi province, China (No 2015KTCL03-06). The authors thank all the nonauthor contributors: Yi Zhen, Yujiao Deng, and Zhe Dou.

\section{Disclosure}

The authors report no conflicts of interest in this work.

\section{References}

1. Siegel RL, Miller KD, Jemal A. Cancer statistics, 2016. CA Cancer J Clin. 2016;66(1):7-30.

2. Jemal A, Siegel R, Xu J, Ward E. Cancer statistics, 2010. CA Cancer J Clin. 2010;60(5):277-300.

3. Mohammed F. Esophageal cancer. $N$ Engl J Med. 2004;350(13): 1363-1364; author reply 1363-1364.

4. Sznol M, Chen L. Antagonist antibodies to PD-1 and B7-H1 (PD-L1) in the treatment of advanced human cancer. Clin Cancer Res. 2013; 19(5):1021-1034.

5. Postow MA, Callahan MK, Wolchok JD. Immune checkpoint blockade in cancer therapy. J Clin Oncol. 2015;33(17):1974-1982.

6. McDermott DF, Atkins MB. PD-1 as a potential target in cancer therapy. Cancer Med. 2013;2(5):662-673.

7. Zou W, Chen L. Inhibitory B7-family molecules in the tumour microenvironment. Nat Rev Immunol. 2008;8(6):467-477.

8. Pardoll DM. The blockade of immune checkpoints in cancer immunotherapy. Nat Rev Cancer. 2012;12(4):252-264.

9. Chen J, Li G, Meng H, et al. Upregulation of B7-H1 expression is associated with macrophage infiltration in hepatocellular carcinomas. Cancer Immunol Immunother. 2012;61(1):101-108.

10. Dong H, Strome SE, Salomao DR, et al. Tumor-associated B7-H1 promotes T-cell apoptosis: a potential mechanism of immune evasion. Nat Med. 2002;8(8):793-800.

11. Lote H, Cafferkey C, Chau I. PD-1 and PD-L1 blockade in gastrointestinal malignancies. Cancer Treat Rev. 2015;41(10):893-903.

12. Parmar MK, Torri V, Stewart L. Extracting summary statistics to perform meta-analyses of the published literature for survival endpoints. Stat Med. 1998;17(24):2815-2834.

13. DerSimonian R, Laird N. Meta-analysis in clinical trials. Control Clin Trials. 1986;7(3):177-188.

14. Mantel N, Haenszel W. Statistical aspects of the analysis of data from retrospective studies of disease. J Natl Cancer Inst. 1959;22(4): 719-748.

15. Baker WL, White CM, Cappelleri JC, Kluger J, Coleman CI; Health Outcomes, Policy, and Economics (HOPE) Collaborative Group. Understanding heterogeneity in meta-analysis: the role of meta-regression. Int J Clin Pract. 2009;63(10):1426-1434.

16. Egger M, Davey Smith G, Schneider M, Minder C. Bias in metaanalysis detected by a simple, graphical test. BMJ. 1997;315(7109): 629-634.

17. Begg CB, Mazumdar M. Operating characteristics of a rank correlation test for publication bias. Biometrics. 1994;50(4):1088-1101.
18. Yuan J, Zhang J, Zhu Y, et al. Programmed death-ligand-1 expression in advanced gastric cancer detected with RNA in situ hybridization and its clinical significance. Oncotarget. 2016;7(26):39671-39679.

19. Wang LA, Wei X, Li Q, Chen L. The prediction of survival of patients with gastric cancer with PD-L1 expression using contrast-enhanced ultrasonography. Tumour Biol. 2016;37(6):7327-7332.

20. Wang L, Ren F, Wang Q, et al. Significance of programmed death ligand 1 (PD-L1) immunohistochemical expression in colorectal cancer. Mol Diagn Ther. 2016;20(2):175-181.

21. Leng C, Li Y, Qin J, et al. Relationship between expression of PD-L1 and PD-L2 on esophageal squamous cell carcinoma and the antitumor effects of CD8(+) T cells. Oncol Rep. 2016;35(2):699-708.

22. Dunne PD, McArt DG, O'Reilly PG, et al. Immune-derived PD-L1 gene expression defines a subgroup of stage II/III colorectal cancer patients with favorable prognosis who may be harmed by adjuvant chemotherapy. Cancer Immunol Res. 2016;4(7):582-591.

23. Dong M, Wang HY, Zhao XX, et al. Expression and prognostic roles of PIK3CA, JAK2, PD-L1, and PD-L2 in Epstein-Barr virus-associated gastric carcinoma. Hum Pathol. 2016;53:25-34.

24. Chen MF, Chen PT, Chen WC, Lu MS, Lin PY, Lee KD. The role of PD-L1 in the radiation response and prognosis for esophageal squamous cell carcinoma related to IL-6 and T-cell immunosuppression. Oncotarget. 2016;7(7):7913-7924.

25. Chen K, Cheng G, Zhang F, et al. Prognostic significance of programmed death- 1 and programmed death-ligand 1 expression in patients with esophageal squamous cell carcinoma. Oncotarget. 2016;7(21): 30772-30780.

26. Böger C, Behrens HM, Mathiak M, Kruger S, Kalthoff H, Rocken C. PD-L1 is an independent prognostic predictor in gastric cancer of Western patients. Oncotarget. 2016;7(17):24269-24283.

27. Zhang L, Qiu M, Jin Y, et al. Programmed cell death ligand 1 (PD-L1) expression on gastric cancer and its relationship with clinicopathologic factors. Int J Clin Exp Pathol. 2015;8(9):11084-11091.

28. Umemoto Y, Okano S, Matsumoto Y, et al. Prognostic impact of programmed cell death 1 ligand 1 expression in human leukocyte antigen class I-positive hepatocellular carcinoma after curative hepatectomy. J Gastroenterol. 2015;50(1):65-75.

29. Tamura T, Ohira M, Tanaka H, et al. Programmed death-1 ligand-1 (PDL1) expression is associated with the prognosis of patients with stage II/III gastric cancer. Anticancer Res. 2015;35(10):5369-5376.

30. Qing Y, Li Q, Ren T, et al. Upregulation of PD-L1 and APE1 is associated with tumorigenesis and poor prognosis of gastric cancer. Drug Des Devel Ther. 2015;9:901-909.

31. Lim YJ, Koh J, Kim K, et al. High ratio of programmed cell death protein $1(\mathrm{PD}-1)(+) / \mathrm{CD} 8(+)$ tumor-infiltrating lymphocytes identifies a poor prognostic subset of extrahepatic bile duct cancer undergoing surgery plus adjuvant chemoradiotherapy. Radiother Oncol. 2015;117(1):165-170.

32. Lim SH, Hong M, Ahn S, et al. Changes in tumour expression of programmed death-ligand 1 after neoadjuvant concurrent chemoradiotherapy in patients with squamous oesophageal cancer. Eur J Cancer. 2016;52:1-9.

33. Kan G, Dong W. The expression of PD-L1 APE1 and P53 in hepatocellular carcinoma and its relationship to clinical pathology. Eur Rev Med Pharmacol Sci. 2015;19(16):3063-3071.

34. Geng Y, Wang H, Lu C, et al. Expression of costimulatory molecules B7-H1, B7-H4 and Foxp3+ Tregs in gastric cancer and its clinical significance. Int J Clin Oncol. 2015;20(2):273-281.

35. Eto S, Yoshikawa K, Nishi M, et al. Programmed cell death protein 1 expression is an independent prognostic factor in gastric cancer after curative resection. Gastric Cancer. 2016;19(2):466-471.

36. Liang M, Li J, Wang D, et al. T-cell infiltration and expressions of T lymphocyte co-inhibitory B7-H1 and B7-H4 molecules among colorectal cancer patients in northeast China's Heilongjiang province. Tumour Biol. 2014;35(1):55-60.

37. Kim JW, Nam KH, Ahn SH, et al. Prognostic implications of immunosuppressive protein expression in tumors as well as immune cell infiltration within the tumor microenvironment in gastric cancer. Gastric Cancer. 2016;19(1):42-52. 
38. Hou J, Yu Z, Xiang R, et al. Correlation between infiltration of FOXP3+ regulatory $\mathrm{T}$ cells and expression of $\mathrm{B} 7-\mathrm{H} 1$ in the tumor tissues of gastric cancer. Exp Mol Pathol. 2014;96(3):284-291.

39. Song M, Chen D, Lu B, et al. PTEN loss increases PD-L1 protein expression and affects the correlation between PD-L1 expression and clinical parameters in colorectal cancer. PLoS One. 2013;8(6):e65821.

40. Shi SJ, Wang LJ, Wang GD, et al. B7-H1 expression is associated with poor prognosis in colorectal carcinoma and regulates the proliferation and invasion of HCT116 colorectal cancer cells. PLoS One. 2013;8(10):e76012.

41. Droeser RA, Hirt C, Viehl CT, et al. Clinical impact of programmed cell death ligand 1 expression in colorectal cancer. Eur J Cancer. 2013; 49(9):2233-2242.

42. Zeng Z, Shi F, Zhou L, et al. Upregulation of circulating PD-L1/PD-1 is associated with poor post-cryoablation prognosis in patients with HBVrelated hepatocellular carcinoma. PLoS One. 2011;6(9):e23621.

43. Loos M, Langer R, Schuster T, et al. Clinical significance of the costimulatory molecule B7-H1 in Barrett carcinoma. Ann Thorac Surg. 2011; 91(4):1025-1031.

44. Wang L, Ma Q, Chen X, Guo K, Li J, Zhang M. Clinical significance of B7-H1 and B7-1 expressions in pancreatic carcinoma. World J Surg. 2010;34(5):1059-1065.

45. Gao Q, Wang XY, Qiu SJ, et al. Overexpression of PD-L1 significantly associates with tumor aggressiveness and postoperative recurrence in human hepatocellular carcinoma. Clin Cancer Res. 2009; 15(3):971-979.
46. Chen XL, Yuan SX, Chen C, Mao YX, Xu G, Wang XY. [Expression of B7-H1 protein in human pancreatic carcinoma tissues and its clinical significance]. Ai Zheng. 2009;28(12):1328-1332. Chinese.

47. Sun J, Xu K, Wu C, et al. PD-L1 expression analysis in gastric carcinoma tissue and blocking of tumor-associated PD-L1 signaling by two functional monoclonal antibodies. Tissue Antigens. 2007;69(1):19-27.

48. Nomi T, Sho M, Akahori T, et al. Clinical significance and therapeutic potential of the programmed death-1 ligand/programmed death-1 pathway in human pancreatic cancer. Clin Cancer Res. 2007;13(7): 2151-2157.

49. Wu C, Zhu Y, Jiang J, Zhao J, Zhang XG, Xu N. Immunohistochemical localization of programmed death-1 ligand-1 (PD-L1) in gastric carcinoma and its clinical significance. Acta Histochem. 2006; 108(1):19-24.

50. Mellman I, Coukos G, Dranoff G. Cancer immunotherapy comes of age. Nature. 2011;480(7378):480-489.

51. Sharma P, Allison JP. The future of immune checkpoint therapy. Science. 2015;348(6230):56-61.

52. Huang B, Chen L, Bao C, et al. The expression status and prognostic significance of programmed cell death 1 ligand 1 in gastrointestinal tract cancer: a systematic review and meta-analysis. Onco Targets Ther. 2015;8:2617-2625.

53. Wu P, Wu D, Li L, Chai Y, Huang J. PD-L1 and survival in solid tumors: a meta-analysis. PLoS One. 2015;10(6):e0131403.
OncoTargets and Therapy

\section{Publish your work in this journal}

OncoTargets and Therapy is an international, peer-reviewed, open access journal focusing on the pathological basis of all cancers, potential targets for therapy and treatment protocols employed to improve the management of cancer patients. The journal also focuses on the impact of management programs and new therapeutic agents and protocols on

\section{Dovepress}

patient perspectives such as quality of life, adherence and satisfaction The manuscript management system is completely online and includes a very quick and fair peer-review system, which is all easy to use. Visit http://www.dovepress.com/testimonials.php to read real quotes from published authors. 